Stephan Pelzl

\title{
An der Grenze von Leben und Tod Euthanasie und Strafrecht
}

Lebensbeendende Maßnahmen mir oder ohne ausdrücklichen Willen des Betroffenen sind nur schwer nach klaren und überzeugenden Kriterien zu regeln. Rechtstheorctisch gesprochen fchlt es an materialen Gesichtspunkten, die trennscharf und einzelfallorientiert zugleich rationale Entscheidungen ermöglichen. Die niederländische Gesetzgebung hat aus dicsem Dilemma einen bemerkenswerten Ausweg gefunden. I992 ist es nach jahrelangen, heftigen Diskussionen gelungen, eine prozedurale Lösung zu entwickeln, die das Verfahren der Nichtverfolgung angemessen und vorhersehbar regelt. Es garantiert, daß nicht cine Person (weder Ärzte noch Angehörige) im "wohlverstandenen Interessc des oder der Sterbewilligen « befugt sein kann, einc irreversible Entscheidung über Leben und Tod zu treffen. Die deutsche Rechtskultur orientiert sich demgegenüber nach wie vor am Modell einer formalisierten und einzelfallbezogenen Dogmatik (System und Kasuistik). Thre Begründungen und Ergcbnisse sind, akzeptiert man dieses Ideal einer wissenschaftlichen Jurisprudenz, erstaunlich differenziert, man denke nur an dic raffinierte Rechtsfindung des „Unterlassens durch positives Tun (etwa beim Abschalten des Beatmungsgeräts). Sie könnte in ihrem Lösungsansatz noch überzeugender werden, wenn die Gesetzgebung eine der widersprüchlichen Vorgaben beseitigen würdc (etwa Streichung des $\$ 2 \times 6 \mathrm{StGB}$ ). Dic Strafbarkeit der Tötung auf Verlangen nach $\$ 216 \mathrm{StGB}$ widerspricht dem Prinzip der Straflosigkcit der Beihilfe zum Selbstmord und dem der Autonomie der Patienten. Die Autoren des Alternativentwurfs zur Sterbehilfe haben daher einen Gesetzgebungsvorschlag gemacht, der auf der Rechtsfolgenseite den Entscheidungsspielraum für Grenzfälle erweitert. Der genannte Entwurf sieht ein Absehen von Strafe (analog zu $₫ 60$ StGB) vor und läßt sich daher aus rechtstheoretischer Perspektive als eine Art Informalisierung der Strafverfolgung cinordnen. Eine Rechtsfolgenentscheidung nach dem Vorbild des $\$ 60$ StGB: Absehen von Strafe durch das Gericht, ermöglicht nämlich bereits der Staatsanwaltschaft, nach $\$ \times 53 b$ StPO ein Strafverfahren einzustellen. Angehörige des Gestorbenen haben dann kcine rechtlichen Möglichkeiten der Klageerzwingung, da es sich um eine Opportunitätsentscheidung handeit. Modelle der Informalisierung setzen also voraus, daß von den Beschuldigten - jenseits materiell-rechtlicher Vorgaben - ein moralisches oder soziales Verständnis für ihre Situation mobilisiert werden kann. Es handelt sich also um Maßnahmen der Diversion. Sie sind daher einer präzisen prozeduralen Regelung der Nichtverfolgung unter rechtsstaatlichen Gesichtspunkten unterlegen. Im folgenden soll vor dem Hintergrund dieser gegensätzlichen kriminalpolitischen Vorgaben die in der deutschen Strafrechtsdogmatik entwickelten Konstruktionen transparent gemacht werden. 
"O Herr, gib jedem seinen eignen Tod, das Sterben, das aus jenem Leben geht, darin er Licbe hatte, Sinn und Not.«'

Für den eigenen, möglichst sanften und schönen Tod existiert seit hellenistischer Zeit vor allem ein Begriff: Euthanasie. Waren dessen Inhalt und Verständnis im Laufe der Jahrhunderte ständigen Veränderungen unterworfen, so stehen wir heute einer Entwicklung gegenüber, welche diesem Wort eine neue Bedeutung verleiht. Die Errungenschaften der modernen Medizin haben ambivalenten Charakter. Eine of tmals zum Selbstzweck gewordene Lebenserhaltung verformt den Sterbeprozeß und nimmt den Menschen am Ende ihres Lebens ihren eigenen Tod. Lebensvcrlängerung wird zur Leidensverlängerung, das Problem des Sterbenmüssens wird um das des Sterbendürfens erweitert.

Mit der medizinisch-technischen Fortentwicklung gehen gesellschaftliche Umbrüche einher. Zu dem Streben nach Lebensqualität gesellt sich der Wunsch nach Sterbensqualität, welcher sich in dem Maße verstärkt, wie die Medizin auch als Bedrohung empfunden wird. Soziale Normen und Wertvorstellungen haben sich gewandelt. Das Verlangen nach Selbstbestimmung auch und gerade am Ende des eigenen Lebens beginnt, die christlich-abendländische Auffassung von der Unverfügbarkeit des menschlichen Lebens zu verdrängen. Den traditionellen Anschauungen stellt sich eine utilitaristische Ethik entgegen, welche die ethische Bewertung einer Handlung allein von den Interessen des Betroffenen abhängig macht. Nicht zuletzt die Zunahme von HIV- und Krebserkrankungen bei gleichzeitigem Anstieg des Bevölkerungsanteils alter Menschen haben dafür gesorgt, daß der Umgang mit dem eigenen Tod Gegenstand öffentlicher Diskussionen geworden ist. Die veränderre Situation an der Grenze von Leben und Tod stellt das Strafrecht vor neue Probleme und verlangt nach Reaktionen.

\section{Der Begriff "Euthanasie"}

Wenn von "Euthanasie« die Rede ist, läßt sich nicht immer eindeutig erkennen, was mit diesem Begriff eigentlich gemeint ist. Oft wird darunter ausschließlich die aktive, geziclt und direkt durchgeführte Tötung eines Menschen zum Zwecke der Beendigung seiner Leiden verstanden ${ }^{2}$. In den angelsächsischen Ländern unterscheidet man zwischen »freiwilliger ", d. h. vom Betroffenen ausdrücklich erbetener, und »unfreiwilliger « Euthanasie. Von letzterer wird bei Menschen gesprochen, die nicht oder nicht mehr fähig sind, einen gültigen Willen zu äußern oder überhaupt zu bilden. Dazu werden beispielsweise Bewußtlose, Schwachsinnige oder Neugeborene gezählt ${ }^{3}$. In den Nicdcrlanden versteht man unter dem Begriff in der Regel die durch einen Dritten herbeigeführte und beabsichtigte Lebensbeendigung eines anderen Menschen aufgrund dessen ausdrücklichen Willens ${ }^{4}$.

I Rilke, Das Stunden-Buch, Drittes Buch, Das Buch von der Armut und vom Tode (1903), 1922, S. 86.

2 So z.B. Engisch, Euthanasie und Vernichtung lebensunwerten Lebens in strafrechtlicher Beleuchtung, 1948, S. 12; Kautzky, in: Eser (Hrsg.), Suizid und Eurhanasie als human- und sozialwissenschaftliches Problem, 1976, S. 287; Langer, JR 1993, 133 (133, 135).

3 Huber, in: Eser/Koch (Hrsg.), Materialicn zur Sterbehilfe, 1991, S. 342.

4 Vgl. Leenen, in: Byrne, (Hrsg.), Medicine, Medical Ethics and the Value of Lifc, 1990, S. 2; van der Wal/van Eijk/Leenen/Spreeuwenberg, Family Practice 1992, Band 9, S. 1 30; Royal Dutch Medical Association, Euthanasia in the Netherlands: The state of the debate, Juni 1993, S. 1. 
Im deutschen Sprachraum wird oftmals der Begriff "Sterbehilfe " verwendet, wohl auch, um sich von dem "Euthanasieprogramm» der Nationalsozialisten abzugrenzen. Das Wort selbst ist jedoch mehrdeutig. Es kann sowohl die Hilfe im oder beim Sterben als auch dic Hilfe zum Sterben umfassen. Hier soll "Sterbehilfe" als die eingebürgerte Übersetzung des gricchischen Wortes verwandt's und die in der deurschen Strafrechtswissenschaft übliche Einteilung des Problemkomplexes im Anschluß an den Psychiater Ehrhardt ${ }^{6}$ übernommen werden.

\section{2. "Echte" Euthanasie}

Führt die geleistetc Sterbehilfe zu keiner Lebensverkürzung, wird teilweise von ciner wechten « oder »reinen « Euthanasie gesprochen”. Sie umfaßt neben seelischer Betreuung auch ärztliche Versorgung bis hin zur Schmcrzlinderung des Todeskampfes. Diese Hilfe im oder bcim Sterben wird oft gar nicht mehr zu dem Bereich der Euthanasie gerechnet ${ }^{8}$, sondern als mitmenschliche Selbstverständlichkeit angesehen. Dic Behandlung zur Schmerzlinderung ist ärztliche Pflicht und kann bei Vernachlässigung zu einer Strafbarkeit des Arztes führen, und zwar wegen vorsätzlicher oder fahrlässiger Körperverletzung durch Unterlassen gem. $\$ \$ 223,230,13$ $\mathrm{StGB}$ oder wegen unterlassener Hilfeleistung nach $\$ 323 \mathrm{C} \mathrm{StGB}$. Für den Bereich der Tötungsdelikte ist die »echte "Euthanasie unerheblich, da sie den Tod nicht vcrursacht. Sie wirft aber viele Problemc hinsichtlich der Rechtspflichten des Arztes gegenüber seinem Patienten auf, die hier nicht näher Gegenstand der Betrachtung sein können.

\section{Passive Euthanasie}

Von denjenigen Formen der Sterbehilfe, die zu einer Verkürzung des Sterbeprozesses beitragen und damit zumindest mitursächlich für den Eintritt des Todes sind, nimmt die sogenannte "passive Euthanasie den in der medizinischen Praxis wohl bedeutendsten Platz ein. Sie umfaßt die I'älle, in denen nichts untcrnommen wird, um den Sterbeprozeß zu verlängern, gegenüber dem man sich also passiv verhält. Es können hierbei drei Arten grob unterschieden werden: die Nichraufnahme einer Behandlung, die Nichtbehandlung einer nach der Therapieaufnahme plötzlich auftretenden (interkurrenten) Krankheit und der Verzicht, eine bereits begonnene Behandlung weiterzuführen". Die letzte Variante wirft noch das Sonderproblem des technischen Behandlungsabbruchs auf. Jeweils sind zwei verschiedene Fallgruppen denkbar. Bei der ersten ist der Patient noch fähig, einen Willen zu bilden und zu äußern, bei der zweiten dagegen ist er dazu nicht oder nicht mehr in der Lage. In jedem Fall trifft man auf die Frage, wo die Grenzen ärztlicher Behandlungspflicht liegen und inwieweit das medizinisch Machbare zur Lebensverlängerung eines Menschen eingesetzt werden darf oder gar muß.

s Kritisch dazu Sporken, in: Eser (Hrsg.) (Fn. 2), S. 271, 273.

6 Euthandsic und Vernichtung slebensunwerten Lebens, Forum der Psychiatrie Nr. 11, 1965, S. sf.

7 Hanack, in: Hiersche (Hrsg.), Euthanasie. Probleme der Sterbehilfe. Eine interdisziplinare Stellungnahmn, 1975, S. 122 ; Schroeder, in: Maurach/Schroeder/Maiwald, Strafrecht BT 1, 7. Auflage 1988, $\$ 1$, Rdnr. 36; Schrciber, NStZ 1986, 337 (338)

8 So Rudolf Schmit, JZ 1979, $462(463)$. 
Dic crste Fallgruppc läßt sich als »cinverständlichen « Behandlungsverzicht bezeichnen. Der Arzt handelt hier auf ausdrücklichen Wunsch des Patienten, wenn er davon absieht, Maßnahmen zur Therapie aufzunehmen oder weiterzuführen. Es geht dabei um den Stellenwert und das Verhältnis des Patientenwillens zu der Behandlungspflicht des Arztes, der für seinen Patienten rechtlich als Garant im Sinne des $\$ 13$ StGB einzustehen und grundsätzlich das ihm Mögliche zur Frhaltung dessen Lebens zu unternehmen hat. Kommt er dieser Pflicht nicht nach, macht er sich eines vorsätzlichen oder fahrlässigen Tötungsdeliktes durch Unterlassen schuldig. Geht man mit der Rechtsprechung davon aus, daß die ärztliche Heilbehandlung den Tatbestand der Körperverletzung erfüllt, so bedarf ihre Durchführung immer der rechtfertigenden Einwilligung des Kranken. Nach dieser Konstruktion ist jedes Tätigwerden des Arztes auf der Tatbestandsebene des $\ 223$ StGB an den Patientenwillen gebunden. Ein Versagen der Einwilligung ist selbst dann bindend, wenn dies grob unvernünftig ist und der Tod des Kranken unweigerlich einzutreten droht. Die eigenmächtige Heilbehandlung ist in jedem Fall als Körperverletzung strafbar ${ }^{10}$.

Voluntas, nicht salus aegroti suprema lex. Dicses Prinzip beansprucht auch im Bereich der Sterbehilfe Gültigkeit. Wird der Patient zu einem bloßen Objekt der technisierten Intensivmedizin herabgewürdigt, kann er seinen Willen als Korrektiv dagegen setzen. Durch Art. 2 Abs. 2 Satz I GG ist zudem ein solches Selbstbestimmungsrecht verfassungsrechtlich abgesichert' '. Der Arzt ist weder verpflichtet noch ist es ihm rechtlich erlaubt, gegen den Willen eines Sterbenden lebensverlängernde Maßnahmen durchzuführen. Nicht der dicsen Willen respektierende Arzt handelt pflichtwidrig, sondern derjenige, der sich darüber hinwegsetzt ${ }^{\prime 2}$. Der einverständliche Behandlungsverzicht ist demnach straflos. Problematisch bleibt allenfalls, wo die Grenzen der ärztlichen Aufklärungspflicht liegen und welche Voraussetzungen der Wille des Patienten erfülien muß, um als beachtlich gelten zu können. Hierbei bictet sich eine Orientierung an dem ausdrücklichen und ernstlichen Verlangen im Sinne des $\$ 2$ I 6 Abs. I StGB an. Auch könnten diejenigen Kriterien Anwendung finden, nach denen ein Selbsttötungsentschluß als frei- oder eigenverantwortlich anzusehen ist's.

Engisch meint, damit sei hinsichtlich des einverständlichen Behandlungsverzichts das Problem der passiven Euthanasie gelöst ${ }^{14}$. Doch hicrbei ist zweierlei zu bedenken. Erstens ist es dogmatisch nicht einfach, eine Straffreiheit zu begründen. Zweitens lassen die vom Bundesgerichtshof im Wittig-Urteil's aufgestellten Thesen kaum noch Raum für ein einverständliches Sterbenlassen. Das Selbstbestimmungsrecht des Patienten ist dort nur ein Abwägungsfaktor innerhalb ciner eigenverant-

9 Vgl. dazu und zu wetteren Unterscheidungsmöglıchkeiten Eser, in: Eser (Hrsg.) (Fn. 2), S. S.

10 Geilen, Euthanasie und Selbstbestimmung, Juristische Betrachtungen zum "Recht au den eigenen Tod $\alpha$, 1975, S. 8; RGSt 25, 375 (382); BGHSt 11, 111 (113 f.); st. Rspr.

11 BVer\{GE $\{2,131(x 70)$; BGHS $11,111(114)$.

12 Engisch, in: Fser (Hrsg.) (Fn. 2), S. 317 f.; Jahnke, in: LK, St rafgeserzbuch, 10. Auflage 1989, vor $\$ 211$, Rdnr. 13; Otto, Gutachten D fur den 56. Deutschen Juristentag Berlin 1986, S. 38; liser, in: Schónke/Schroder (Sch/Sch), Strafgesetzbuch, 24. Auflage 199r, vor $\$ \$ 2 \times 1$ If., Rdnr. 28.

$r_{3}$ Diese sind jedoch hefig umstritten; vgl. dazu Neumann, JA 1987,244 fi., m.w.N.

14 Engisch, in: Eser (Hrsg.) (Fn. 2), S. 316.

is BCHSt 32, 367: Der angeklagte Hausarzt Dr. Witrig fand seine 76 Jahre alte Patientin in ihrer Wohnung bewußtlos vor, nachdem sie eine Überdosıs Morphium und Schlaftabletten zu sich genommen hatte und nicht ohne schwere Dauersehaden zu retten gewesen ware. Sie hatte d.anit die schon fruher mundlich wie schriftlich geäußerten Selbstmordabsichten verwirklichen wollen. Der Arzt leeßs sic sterben und wurde in leczter Instanz freigesproehen. Die Begrundung des BGH ist un der Lehre auf Kritik gestoßen; vgl. Eser, MedR 1985, $6 \mathrm{ff}$. und Rudolf Schmitr. JZ 1985 , 365 (367 f.); dagegen Herzberg, JA 1985, 117 (184f.), $26 \mathrm{~s}$ ( $267 \mathrm{ff}$.); Kutzer, MDR 1985,710 (711 ff.) 
wortlichen Entscheidung des Mediziners. Das kommt einer Entmündigung des Patienten nahe, denn cin Selbstbestimmungsrecht, über das andere bestimmen, ist keines mehr. Wenn der Sterbewille unbeachtlich ist und dic Entscheidungsbefugnis auf den Arzt übcrgeht, kann von einem »einverständlichen« Sterbenlassen nicht mehr die Rede sein ${ }^{16}$.

\section{b) Einseitiger Behandlungsverzicht}

Ist der Patient nicht oder nicht mehr in der Lage, seinen Willen zu äußern und so die Behandlungspflicht des Arztes cinzuschränken, ist zu fragen, nach welchen Kritcrien ein "einseitiger" Behandlungsverzicht durch den Mediziner erfolgen darf. Da seit Endc der scchziger Jahre der Hirntod als Todeszeitpunkt in Recht und Medizin anerkannt ist, wird jedem ärztlichen Tun spätestens dann eine Grenze gezogen, wenn das Gehirn des Patienten vollständig abgestorben ist ${ }^{17}$. Die Frage nach der rechtlichen Zulässigkeit eines Behandlungsverzichts ohne den Willen des Betroffenen werfen nun vor allem diejenigen Fälle auf, bei denen zwar das Großhirn abgestorben ist, das Stammhirn und damit die vegetativen Funktionen aber noch erhalten sind (apallisches Syndrom). Solche "A palliker « atmen, verdauen, schwitzen, können aber mit ihrer Umwelt in keiner Weise in Verbindung treten.

Grundsätzlich ist man sich zwar einig, daß die ärıtliche Behandlungspflicht auch schon vor Eintritt des Gesamthirntodes begrenzt wcrden kann ${ }^{18}$. So hat der Bundesgerichtshof in seinem ansonsten umstrittenen Wittig-Urteil ausgeführt: "Andererseits darf der Arzt berücksichtigen, daß es keine Rechtsverpflichtung zur Erhaltung eines erlöschenden Lebens um jeden Preis gibt. Maßnahmen zur Lebensverlängerung sind nicht schon deswegen unerläßlich, weil sie technisch möglich sind. «" Wenn es aber darum geht, Kriterien für eine Begrenzung aufzustellen, so »betritt man mit dem reinscitigen Behandlungsabbruch völlig ungcsichertes Gelände « ${ }^{20}$. Vielfach begnügt man sich mit allgemeinen Äußerungen und wenig präzisen Formulierungen. Als Abbruchsgrund ist zwar der unwiderrufliche Bewußtseinsverlust allgemein anerkannt, hinsichtlich der Begründung bestehen aber keinc gesicherten Erkenntnisse $^{11}$. So wird ausgeführt, die Menschenwürde des im Koma Liegenden gebicte es, auf eine weitere Behandlung zu verzichten ${ }^{22}$. Der ärztliche Auftrag sci hier beendet und eine Weiterbehandlung unzumutbar ${ }^{23}$ oder, wie im medizinischen Schrifttum verbreitet, sinnlos ${ }^{24}$. Auch wird auf dic Schicksalhaftigkeit und die Natürlichkeit des Todes hingewiesen"s und zwischen "gewöhnlichen " und "außergewöhnlichen « Maßnahmen unterschieden ${ }^{26}$. Dancben wird noch ein Behandlungsver-

16 Fser, MedR 1985,6 ( 13$)$.

17 Hanack (Fn. 7), S. 144; Arthur Kaufmann, JZ. 1982, 481 (486).

18 Jahnke (Fn. 12), Rdnr. 17 F.; Otto (Fn. 12), S. so; a. A. lediglich Bockelmann, Strafrecht des Arztcs, 1968 , S. 31, Anm. 38: Aber wenn der Arzt überhaupe die Pflicht zu helfen ubernimmt, so kann diese Pflicht nur einen Inhalt haben, namlich den, daß er unter allen Umstanden helfen muß, soweit nur seine Moglichkeiten reichen."

I9 BGHSt 32, 367 (3791.).

20 Eser, JZ $1986,786(792)$

21 Ebenda, 793.

22 V. Dellinghausen, Stcrbchilfe und Grenzen der Lebenserhaltungspflichr des Arz.tes, 198 1, S. 401 f.; F.ser, in: Auer/Menzel/Eser, Zwischen Heslauftrag und Sterbehilfe, 1977, S. 147; Moliering, Schutz des Lebens - Recht auf Sterben. Zur rechtlichen Problematik der Euthanasic, 1977, S. $56 \mathrm{ff}$.

23 Escr, in: Sch/Sch, vor $\$ 21$ i fl., Rdnr. 29; A rthur Kaufmann (Fn. 17), 485; v. Lutterotri, Mcnschenwurdiges Sterben, 1985, S. $58 \mathrm{f}$.

${ }_{24}$ Friciche, in: Eser (Hrsg.) (Fn. 2), S. 153 ; v. Lutterotti (Fn. 23), S. 291 ; Opderbecke, in: ebenda, S. 137.

25 Auer, in: Eser (Hrsg.) (Fn. 2), S. 250 ff.; Möllering (li11. 22), S. 59 ff.; Sax, JZ 1975, 137 (141 f., 149).

26 Ziegler, in: Hiersche (Hrsg.) (Fn. 7), S. 77, sowie dic Katholische Kirche vor allem seit Papst Pius XII; vgl. auch Hans Jonas, Technk, Medizin und Ethik . Zur Praxis des Prinzips Verantwortung, 2. Auflage 1987 , S. $261 \mathrm{f}$ 
zicht auf der Grundlage einer mutmaßlichen Einwilligung ${ }^{27}$, eines »Patiententestamentes« oder einer Vertretung durch die Angehörigen diskutiert. Ungeklärt ist auch, inwieweit der ärztlichen Behandlungspflicht ökonomische Grenzen gesetzt werden können, und wie ein selektiver Einsatz begrenzter medizinischer Kapazitäten rechtlich zu beurteilen ist ${ }^{28}$.

Fehlt es an einem ernstlichen und ausdrücklichen Willen des Patienten, liegt es nahe, dessen "mutmaßliche« Einwilligung als Kriterium für weitere Behandlungen heranzuziehen. Hierbei wird jedoch zu beachten sein, daß dieser lediglich hypothetische Wille nicht zwingend alleiniger Maßstab sein kann. Wenn ein früher geäußerter Wille, auch bei irreversiblem Bewußtseinsverlust in jedem Falle unendlich lange am Leben erhalten zu werden, einem Behandlungsabbruch nicht entgegen steht ${ }^{29}$, kann auch der erklärte Wunsch, bei dauerhaftem Koma sterben zu dürfen, nicht zwangsläufig zur Zulässigkeit des Behandlungsabbruchs führen. Zunächst kommt der Frage zentrale Bedeutung zu, inwieweit das Großhirn zerstört ist, ob also der Bewußtseinsverlust ein dauerhafter sein wird. Dies ist oftmals nicht eindeutig bestimmbar. Gerade bei Apallikern wird von Fällen berichtet, in denen deren Zustand ganz oder teilweisc reversibel gewesen ist ${ }^{30}$. Wenn nach medizinischem Ermessen keine Aussicht besteht, daß der Patient auch nicht einen Teil seines Bewußtseins jemals wiedererlangen kann, sollte ein Behandlungsverzicht zulässig und gerechtfertigt $\operatorname{sein}^{31}$. Die passive Sterbehilfe wäre dann lediglich eine Korrektur des medizinischtechnischen Fortschritts, ohne den der Patient schon gestorben wäre.

In Zweifelsfällen bleibt die Frage, ob sich nicht ein mutmaßlicher Wille ermitteln läßt, dem dann eine den Therapieverzicht rechtfertigende Wirkung zukäme. Es könnten Angehörige nach früheren Äußerungen des Patienten befragt werden oder "Patiententestamente "Anhaltspunkte liefern ${ }^{32}$. Eine Vertretung durch Angehörige ist dagegen abzulehnen, da eine Entscheidung über das eigenc Leben immer nur eine höchstpersönliche sein kann und die Gefahr bestünde, daß Fremdinteressen über Leben und Tod eines anderen entschieden. Besteht für den Patienten noch die Möglichkeit, das Bewußtsein zumindest vorübergehend oder nur teilweise wiederzuerlangen, darf ein einseitiger Behandlungsverzicht nicht vorgenommen werden. In jedem Fall sollte dem behandelnden Arzt lediglich ein Beurteilungs- und kein alleiniges Entscheidungsermessen zukommen. Vor Therapieverzicht ist zumindest $\operatorname{cin}$ weiterer Arzt hinzuzuziehen. In der Regel wird die Entschcidung auch nicht von einem einzelnen Mediziner getragen, sondern erfolgt im Rahmen eines interdisziplinären Konsiliums und begründet sich auf den Konsens der beteiligten Ärzte ${ }^{33}$. Wichtig dabei ist, mittels eincr Dokumentationspflicht für Transparenz und Nachprüfbarkeit zu sorgen, um einem möglichen Mißbrauch in einer rechtlichen Grauzone auf den Intensivstationen der Krankenhäuser Einhalt zu gewähren.

27 Zuletzt BGH JR 1993,167 (169).

28 Aut diese Probleme kann hier niche nàher :ingegangen werden. Vgl. zum ersten Punkt Opderbecke, MedR 1985, 23 (27 ff.); zum zweiten Kuper, JuS 1981, 78, ff. sowic durs., JuS 1971, 474 (476).

29 Otuo (Fn. 12), S. 36.

$30 \mathrm{Vgl}$. v. Lutcerotu (Fn. 23), S. $82 \mathrm{f}$

31 Merkel, in: Hegselmann/Merkel (Hrsg.), Zur Debacte uber Euthanasic, 1991, S. 98 f., sieht den irreversiblen Bewußtseinsverlust als die alleinige Grundlage fur einen Behandlungsverzicht an, da es dann an einem Lebensinteresse des Patienten fchlte.

32 Für eine Indizwirkung auch Dreher/Trondle, Strafgesetzbuch, 45. Auflage 1991, vor $§ 211$, Rdnr. 18 ; Eser, in: Auer/Menzel/Eser (Fn. 22), S. $x_{4} 4$.

33 Opderbecke (Fn. 28). 
Die oben skizzierten Begründungen für die Zulässigkeit der passiven Euthanasie lassen ihre "Regelungsmängel ${ }^{34}$ deuclich werden, wenn es um deren dogmatische Einordnung geht. Das gilt sowohl für den einverständlichen wie auch den einseitigen Behandlungsverzicht und vor allem dann, wenn man sich der Grenze zum positiven Tun nähert. Zunächst ist es konsequent, die Garantenpflicht des Arztes durch das Prinzip voluntas aegroti suprema lex zu begrenzen. Wenn man dem Patienten gestattet, seinen Willen gegen den Behandlungsbeginn zu setzen, so muß man cs ihm auch erlauben, die Fortführung einer bereits begonnenen Therapie zu verweigern. Ansonsten zwänge man Arzt und Paticnt »zu dem heuchlerischen Vorbehalt...., jeweils nur den nächsen Schritt vereinbart zu haben, um den übernächsten dann schlicht verweigern zu können «" 35 . Doch werden hier erste Wertungswidersprüche erkennbar. So ist zum einen der Wille des Patienten schlechthin bindend und die eigennächtige Heilbehandlung als Körperverletzung strafbar, auch wenn die Verweigerung der Einwilligung grob unvernünftig ist und ein tödlicher Ausgang droht. Dagegen führt selbst ein noch so vcrständliches Todesverlangen des Kranken zur Strafbarkeit wegen Tötung auf Verlangen, wenn der Arzt dem Wunsch nachkommt ${ }^{36}$. Dieses Mißverhältnis ist insoweit berechtigt, weil sich der Sterbewunsch im Fall des $\$ 216$ StGB an einen Dritten wendet, für den jenes Prinzip eben nicht gelten kann. Das Selbstbestimmungsrecht findet seine Grenze dort, wo es einer anderen Person zu seiner Verwirklichung bedarf ${ }^{37}$.

Dicse Vorschrift wirft jedoch ein anderes Problem auf. Der behandelnde Arzt als Garant kann nur dann straffrei sein, wenn scine Garantenpflicht durch die (mutmaßliche) Einwilligung des Patienten beseitigt wird. Unbestritten stellt $\$ 216$ StGB jedoch klar, daß die Einwilligung des Getöteten niemals die Tötungshandlung eines Dritten rechtfertigen kann $n^{38}$. Von dem Rechtsgrundsatz "volenti non fit iniuria wird hier abgewichen. Bindet man nun die Garantenpflicht des Arztes an den Patientenwillen, so umginge man diese Sperre wieder. Deshalb kann das, was für das disponible Rechtsgut der körperlichen Integrität gilt, nicht von vornherein auch für das Rechtsgut Leben gelten ${ }^{39}$. Als Ausweg wird angenommen, \216 StGB könne nicht oder nur sehr eingeschränkt durch Unterlassen verwirklicht werden ${ }^{40}$. Dies wiederum setzt voraus, daß die der passiven Euthanasie zugeordneten Verhaltensweisen vom bloßen Untätigbleiben bis zum »aktiven « Abschalten einer Maschine auch rechtlich als Unterlassen zu werten sind.

Die Nichtaufnahme einer Behandlung sowie der Verzicht auf weitcre Maßnahmen lassen sich recht unproblematisch als Unterlassen einordnen. In beiden Fällen bleibt der Arzt untätig. Die Beschleunigung des Todeseintritts wird anderen, von außen kommenden Faktoren überlassen. Oft wird eine zusätzlich auftretende Lungenentzündung nicht bekämpft. Anders ist dies beim sogenannten technischen Behandlungsabbruch. Hier muß ein Dritter erst tätig werden, um den Tod beispielsweisc

34 Eser, JZ 1986, 787 (793).

3s Ders., in: Auer/Menzel/Eser (Fn. 22), S. 107 f.

36 Hinzu kommt noch, daß die sBeihilfe $\alpha$ zum Selbstmord, also das Reichen des Stricks oder Giftbechers, von Einzelmeinungen abgesehen straflos ist.

37 Engisch, in: Eser (Hrsg.) (Fn. 2), S. $319 \mathrm{f}$.

38 Vgl. Jähnke, in: LK, $\$ 216$, Rdnr. 17; Eser, in: Sch/Sch, $\$ 216$, Rdnr. 13 ; Schroeder (Fn. 7), $\$ 2, R d n r .62 ;$ BGHSt $4,88(93)$.

39 Eser, in: Sch/Sch, vor $\$ 211 \mathrm{ff}, \mathrm{Rdnr} .28$.

40 Ders., in: Sch/Sch, $\$ 216$, Rdnr. 10; Horn, in: SK, Strafgesetzbuch, 4. Auflage, 29. Lieferung (Srand Juni 1991), $\$ 2$ 16, Rdnr. 14; Archur Kaufmann, MedR 198 3, 121 (1 22); Sax (Fn. 25), 146, Anm. 81 ; a. A. Herzberg, NJW 1986, 163 s (1638); Rudolf Schmitt, MDR 1986, 617 (620); BGHSt 13, 162 (166); 32, 367 (370). 
durch Abschalten des Reanimators herbeizuführen. Man ist sich nicht cinig, ob dieses Tätigwerden als Unterlassen im Rechtssinn anzusehen ist. Unstrittig ist lediglich das Ergebnis. Der technische Behandlungsabbruch ist nach den gleichen Kriterien wie der sonstige Therapieverzicht grundsätzlich erlaubt ${ }^{4}$.

So cinheitlich die Zulässigkeit auch angenommen wird, so uneinheitlich sind die Begründungen dafür. Überwiegend wird vertreten, der Abbruch einer apparativen Behandlung sei aufgrund des sozialen Sinngehaltes des Verbaltens oder mittels der Rechtsfigur des "Unterlassens durch Tun " $^{4^{2}}$ als Unterlassen im Sinne des $\$$ I $_{3} \mathrm{StGB}$ zu werten ${ }^{43}$. In der Tat scheint dieser Weg der einfachere zu sein. Besteht keine Behandlungspflicht mehr, unterläßt der Arzt Maßnahmen, zu denen er nicht verpflichtet ist, einc strafrechtlichc Handlung scheidet aus. Betrachtet man zudem die Funktion der lebenserhaltenden Gcräte selbst, so werden durch sie nur viele Einzelakte manueller ärztlicher Handlungen ersetzt, deren Nichtvornahme zweifelsfrei ein Unterlassen darstellen 44 . Die jcweilige Apparatur wäre quasi der verlängerte Arm dcs Arztes ${ }^{45}$, dessen aktive Fürsorge durch das Abschalten in ein passives Geschehenlassen mit ungewissen Folgen überginge ${ }^{46}$. Diese an die soziale Handlungslehre anknüpfende Sichtweise muß allerdings in Fällen problematisch werden, bei denen ein bloßer Knopfdruck nicht mehr ausreicht, um das gewünschte oder zumindest akzeptierte Ergebnis, den Tod des Patienten, herbeizuführen. Phänotyp und juristische Einordnung fielcn so immer deutlicher auseinander, die Grenzen zur aktiven direkten Euthanasie drohten zu verschwimmen. Die Rechtsfigur des „Unterlassens durch Tun « sieht sich schließlich dem Einwand ausgesetzt, in allzu durchsichtiger Weise die Lebensverkürzung in ein Unterlassen einer Sterbensverlängerung umzudeuten ${ }^{47}$.

Diejenigen, die auf ein positives Tun abstellen ${ }^{48}$, sehen sich dagegen zu "konstruktiven Höchstleistungen mit buchstäblich Orwell'schen Visionen $\star^{49}$ veranlaßt, um eine Straffreiheit herzuleiten. So wird das "Recht auf einen nnatürlichen Tod«" angeführt ${ }^{\text {so }}$ oder die Straffreihcit damit bcgründet, das verlöschende Lcben sei nicht mehr vom Schutzzweck der Norm erfaßt". Auch ist zu lesen: "Weil es in dieser Situation um dic Herstellung der 'Behandlungsfreiheit، gcht, die die Achtung des verfassungsrechtlich garantierten Selbstbestimmungsrechts der Person, Art. I, 2 Abs. 2 S. I GG, gewährleistet, sind die zur Verwirklichung des verfassungsgemäßen Zustandes nötigen Maßnahmen gerechtfertigt. «sz

Die Zulässigkeit des technischen Behandlungsabbruchs sollte nicht von der Einordnung als Tun oder Unterlassen abhängen. Ein solches "Kategoriengeklapper «s3 stößt bei vielen Medizinern zu Recht auf Unverständnis. Es kann eigentlich keinen Unterschied machen, ob der Sterbeprozeß durch Abbruch einer Therapie, durch Nichtbehandlung einer interkurrenten Krankheit oder durch Abschalten technischer

41 Vgl. dazu Eser, in: Sch/Sch, vor $\$ 211, R d n r .32 ;$ Jahnke, in: LK, $\$ 216, R d n r .13 ;$ vor $\$ 211, \operatorname{Rdnr} 16$. 42 Roxin, in: Bockelmann/Arthur Kaufmann/Kluy (Hrsg.), Festschrift fur Engisch, 1969, S. 38 .

43 Engisch, in: Jescheck/Luttger (Hrsg.), Festschrift fur Dreher, 1977, S. 329 ; Eser, in: Auer/Menzel/Eser (Fn. 22), S. 140; Hanack, a.a. O. (Fn. 7), S. 145; Roxin (Fn. 42), S. 395 ff.; ders., NStZ 1987, 345 (349). 44 Hanack (Fn. 7), S. 145

45 So insbesondere Geilen (Fn. 10), S. 22, Anm. 36.

46 Engisch, in: Jescheck/Luttger (Hrsg.) (Fn. 43), S. 327

47 Sax (Fn. 25), 142.

48 Bockelmann (Fn. 18), S. 112; Hirsch, in: Kúper (Hrsg.), Festschrift lur Lackner, 1987, S. 605; Jahnke, in: LK (Fn. 41); Jescheck Strafreche AT, 4 . Auflage 1988, S. \$46; Otto (Fn. 12), S. 43; Samson, in: Stratenwerth u. a. (Hrsg.), Festschrift Für Welzcl, 1974, S.60r; Sax (Fn. 25), 137

49 Eser, JZ 1986, 786 (793).

so Samson (Fn. 48), S. 602.

s1 Jähnke (Fn. 41), Rdnr. 17; Sax (Fn. 25), 144 ff.

$\{2$ Otto (Fn. 12), S. 4 s.

53 Engisch (Fn. 43), S. 315 . 
Apparatc beschleunigt wird, welche ja nur Teil einer Behandlung sindst. Das faktische Umdeuten eines positiven Tuns in ein Unterlassen erlaubt es, die Grenze zwischen strafloser passiver und strafbarer aktiver Sterbehilfe relativ einfach und eindeutig aufrecht zu erhalten. Dazu schreibt Engisch: »Hier bietet sich dann die sMogelei an, statt durch ein aktives Tun... durch ein das ,Tabu respektierendes Unterlassen... das Lebensende herbeizuführen. «ss Damit ist es möglich, eine als »sinnlos" erachtete Lebens- bzw. Sterbensverlängerung zu vermeiden und gleichzeitig das Prinzip aufrecht zu erhalten, jede aktive Sterbehilfe sei unzulässig. So wird die Problematik ausschließlich auf die Frage beschränkt, wann ein Weiterbehandeln medizinisch nicht mehr sinnvoll ist. Doch zu fragen ist eher, "wo das Postulat nach formalisierterem und unbegrenztem Lebensschutz in Inhumanität umschlägt s $^{66}$, wo der medizinische Fortschritt eines ethischen Korrektivs bedarf. Der Jurist darf sich nicht auf eine medizinische Ebene bei einer Frage flüchten, die in erster Linie ihn betriffts?.

Das Problem läßt sich auch nicht mit dem »richtig verstandenen Schutzbereich der Norm ${ }^{58}{ }^{8}$ lösen, da dann die Reduzierung der Normgeltung grundsätzlich auch für die direkte Euthanasie gelten müßte. Ist das verlöschende Leben nicht mehr vom Schutzzweck der Tötungsdelikte erfaßt, gäbe man damit zu erkennen, daß es wegen seines Zustandes weniger schutzwürdig ist ${ }^{s 9}$. Jedoch muß auch verlöschendes Leben prinzipicll geschützt werden. Eine Lösung sollte jedenfalls nicht auf der Tatbestandsebene gesucht werden ${ }^{60}$. Damit entschiede auch die Einordnung des technischen Behandlungsabbruchs als Tun oder Unterlassen nicht mehr über Strafbarkeit oder Straffreiheit, was dem Grenz- und Übergangscharakter dieses Verhaltens angemessen wäre ${ }^{6 !}$. Insgesamt ist es mit dem geltenden Recht nicht möglich, für die passive Euthanasie eine Regelung zu finden, die frei von Widersprüchen ist. Solange nicht der gesamte Bereich der lex artis ausgeführten ärztlichen Eingriffe und Maßnahmen einer sachgerechten Lösung zugeführt ist, wird man sich mit den gegenwärtigen Unzulänglichkeiten abfinden müssen ${ }^{62}$. Es besteht daher nach wie vor ein Rcgelungsbedarf für den Gesetzgeber, ein Bedarf, der mit unaufhaltsamer technischer Fortentwicklung bei knapper werdenden finanziellen Mitteln um so größer werden wird, will man nicht die Entwicklung in den Intensivstationen sich selbst überlassen.

\section{Indirekte Euthanasie}

Besteht das oben angesprochene "Tabu « darin, jede aktive Lebensverkürzung sei ohne Ausnahme unzulässig ${ }^{6}$, bedeutet dic Sonderbehandlung der sogenannten »indirekten « Euthanasie einen „Einbruch in das traditionelle Verbot der saktiven Euthanasie « ${ }^{64}$. Zu dieser Art der Sterbchilfe werden diejenigen Lebensverkürzungen

\$4 Eser, in: Auer/Menzcl/Eser (Fn. 22), S. 139.

ss Engisch (Fn. 43); Hervorhebungen im Original.

s6 Samson (Fn. 48), S.602 f.

57 Ebenda.

s\$ Jähnke (Fn. \$1).

S9 Eser, in: Aucr/Menzel/Eser (Fn. 22), S. 140.

6o Der Alternativentwurf Sterbehilfe sicht e'me besondere Rechtfertigungsmoglichkeit vor; vgl. Baumann u. a. (Hrsg.), Alternativentwurf eines Gesetz.s's uber Sterbehilfe, 1986, $\$ 214$.

6. Kuper, JuS 1971, 474 (477); er schlagt einen übergesetzlichen entschuldigenden Notstand vor.

62 In diesem Sinne Dreher/Trondle, vor $\$ 2: 1$, Rdnr. 17; siche auch Trondle, ZStW 99 (1987), 25 (33 ff.).

63 So ausdrucklich Jahnke, in: LK, vor $\$ 211, R d n r .14$.

64 Hanack (Fn. 7), S. 146; auch Jahnke (Fn.63), Rdnr. is, bchandele die indirekte Euthanasie gesondert. 
gezählt, dic nur unbeabsichtigtes Behandlungsrisiko einer schmerzstillenden Therapie unter Inkaufnahme des möglichen vorzeitigen Todes sind ${ }^{6 s}$. Wic bei der passiven Sterbehilfe ist zu beobachten, daß man sich im Ergebnis weitgehend einig ist, während die Begründungen für die Straflosigkeit völlig umstritten sind. Sicher ist nur, daß schmerzlindernde Maßnahmen, die zu eincr Lebensverkürzung führen, Tötungshandlungen im Sinne der Tötungsdelikte sind. Im Einzelfall kann es allerdings problematisch sein, den Beweis des erfolgskausalen Tuns zu erbringen, da jede Schmerzlinderung auch einen Einfluß auf den Gesamtorganismus hat und unter Umständen das Leben eher verlängert als verkürzt ${ }^{66}$. Unerheblich für die Strafbarkeit ist damit eine Garantenpflicht. Wie für jede andere ärztliche Maßnahme bedarf auch eine Schmerztherapie der Einwilligung des Patienten, vor allem dann, wenn die Möglichkeit einer Lebensverkürzung besteht. Fehlt es an der Einsichts- und Urteilsfähigkeit des Kranken, ist im Zweifel davon auszugehen, daß die Schmerzlinderung in seinem Interesse liegt und von eincr mutmaßlichen Einwilligung gedeckt ist.

\section{a) Dogmatische Begründungen}

Die dogmatischen Begründungen für die Zulässigkeit der indirekten Eurhanasie vcrteilen sich auf alle Deliktsebenen. Teils wird der Tötungsvorsatz verneint ${ }^{67}$, teils wird vertreten, die lebensgefährliche Schmerzlinderung werde nicht mehr vom Schutzzweck der Norm erfaßt ${ }^{68}$. Auf der Rechtfertigungsebene wird das erlaubte Risiko ${ }^{69}$ sowie der Notstand nach $₫ 34$ StGB angeboten ${ }^{\circ}$. Schlicßlich wird noch angenommen, ein Schuldvorwurf sei ausgeschlossen ${ }^{\text {". }}$.

Verfchlt ist der Ansatz, welcher den Tötungsvorsatz verneint, da es der Rechtsklarheit wegen eines objektiv vertretbaren Weges bedarf ${ }^{72}$. Zudem wird der Vorsatz in der Regel vorliegen, da es für diesen im wesentlichen nur auf die Kenntnis der Folgen einer Handlung ankommt. Der gut über die Möglichkeiten einer Therapie und ihre Nebenwirkungen informierte Arzt wird sich auch über die Risiken und damit über eine drohende Lebensverkürzung bewußt sein. Wenn er dies crkannt hat, ist es für den Vorsatz gleichgültig, ob er die lebensverkürzende Wirkung nur als Nebenfolge ansieht oder nicht. Er wird in jcdem Fall zumindest dolus eventualis haben ${ }^{73}$.

Fraglich crscheint, ob man den Schutzzweck der Tötungsdelikte deshalb begrenzen kann, weil Maßnahmen zur Schmerzlinderung unter Inkaufnahme einer Abkürzung des Lebens "ihrem sozialen Gesamtsinn nach aber etwas ganz anderes als eine >Tötungshandlung< i.S. des $\$ 2 \times 2 \alpha^{74}$ sind. Das hieße, dem verlöschenden Leben die Schutzwürdigkeit gegenüber ciner Verkürzung grundsätzlich abzusprcchen75. Daneben ist diese Lösung auch unzureichend, da sie sich auf das Endstadium einer tödlichen Krankheit beschränken müßte, eine Schmerzlinderung mit dem Risiko der

6s Ehrhardt (Fn. 6), S. sf.; Jahnke (Fn. 63), Rdnr. 12, 15; Arthur Kaufmann (Fn. 40); Schreiber (Fn. 7), 340; soweit ersichtlich, liegt zu diesem Bereich bisher noch keine Rechtsprechung vor.

66 v. Lutcerotti, in: Eser (Hrsg.) (Fn. 2), S. 294.

67 Bockelmann (Fn. 18), S. 25, 70: Risikovorsatz ist noch nicht Verletzungsvorsatza.

$68 \mathrm{Krey}$, Strafrecht BT 1, 8. Auflage 199r, Rdnr. 14; ähnlich Blei, Strafrechr BI; 12. Auflage 1983, S. 17; Wessels, Strafrecht BT 1, 16. Auflage 1992, S.7.

69 v. Dellinghausen (Fn. 22), S. 1 iff., 163; Engisch, in: Arthur Kaufmann/Bemmann/Krauss/Volk (Hrsg.), Festschritt fur Bockelmann, 1979, S. 532; Eser, in: Sch/Sch, vor $\$ 211$ Ii., Rdnr. 26

70 Geilen (Fn. 10), S. 26; Hanack (Fn. 7), S. 132 f.; Horn, in: SK, $\$ 212$, Rdnr. 26; Otto (Fn. 12), S. $56 \mathrm{ff}$.; Schreiber (Fin. 7), 340f.

71 Lilufs, Arztrecht, 3. Auflage 1984, Rdrr. 217.

72 Fiser, in: Auer/Menzel/Eser (Fn. 22), S. 88

73 Fbenda; Hanack (Fn. 7), S. 147; Jahnke (Fn. 51).

74 Wessels (Fn. 68).

75 Eser (Fu. 22), S.89. 
Lebensverkürzung jedoch oft schon vorher notwendig werden wird ${ }^{6}$. Will man dagegen lediglich den Schuldvorwurf verneinen, so muß man sich fragen lassen, wieso ein Verhalten, das allgemein gebilligt und medizinisch auch als geboten angesehen wird, der Rechtsordnung zuwider laufen soll. Zudem wäre man wieder im gerade hier problematischen subjekiven Bereich angelangt.

Es bietet sich daher an, den behandelnden Arzt im Falle des Todes seines Patienten nach objektiven Gesichtspunkten zu rechtfertigen"7. Dabei verwischt die Rechtfertigung wegen erlaubten Risikos eher die Grenze zum strafbaren Tötungsdelikt, da man Maßnahmen, die Tötungshandlungen sind, in einen erlaubten Bereich verlagern müßte. Schließlich muß dieser Ansatz spätestens dann versagen, wenn sich das Risiko der Lebensverkürzung zur Gewißheit verdichtet. Viclmehr ist zunächst die Lagc des Arztes zu charakterisieren, um cine angemessene Lösung zu finden. Dieser befindet sich in einem Konflikt zwischen der Pflicht, das Leben seines Patienten nicht zu verkürzen, und der Pflicht zur Schmerzlinderung. Einerseits ist es nach dem Prinzip neminem laedere verboten, zu schaden, den Tod hervorzurufen. Der Hippokratische Eid verpflichtet den Mediziner, in dieser Hinsicht alles zu unterlassen ${ }^{78}$. Andererseits ist es ihm geboten, zu helfen, Leiden zu lindern und Schmerzmittel in den dafür erforderlichen Dosierungen zu verabreichen 79 . Diese Handlungspflicht gerät mit jener Unterlassungspflicht in Konflikt. In solchen Fällen der Pflichtenkollision sind Notstandsgrundsätze maßgebend, ob die an sich verbotene Handlung gerechtfertigt sein kann ${ }^{80}$. Nach dem "Prinzip des überwiegenden Interesses « muß die Lebensverlängerung um eine kurze Frist gegen die unerträglichen Schmerzen abgewogen werden. Das bedarf allerdings zweier Voraussetzungen.

Erstens muß das Rechtsgut Leben überhaupt in den Kreis der abwägbaren Interessen aufgenommen werden. Zweitens ist es notwendig, dieses als nachrangig zu betrachten, so man zur Rechtfertigung kommen möchte. Insbesondere der letzte Punkt ist wohl auch der Grund dafür, daß dieser Ansatz gescheut und zum Teil von vornherein verworfen wird ${ }^{8}$. Dabei wird jedoch zu beachten sein, daß es hier nicht um einc Gegenüberstellung von Interessen verschiedener Personen, sondern von Interessen derselben Person geht. In dem Moment unerträglicher Qualen wird das prinzipielle Lebensinteresse überlagert und verdrängt von dem Interesse an Schmerzlinderung. Sich hier auf die Achtung eines abstrakten Rechtsgutes "Leben « zu berufen und auf eine weitere Schmerzbekämpfung wegen des Törungsrisikos zu verzichten, wäre unmenschlich und auch widersinnig. Man gäbe ja gerade damit die Achtung vor dem konkreten Leben bzw. Sterben des Leidenden zugunsten eines Abstraktums auf $f^{82}$. Eine solche Abwägung im Rahmen des $\$ 34$ StGB ist durchaus

76 Ebenda; Hanack (Fn. 7), S. $133 \mathrm{f}$.

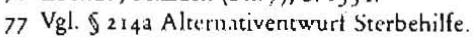

78 sIch werde auch niemandem eine Arznei geben, die den Tod herbeiführt, auch nichs, wenn ich darum gebeten werde, auch nic einen Rat in dieser Richtung erteilen. $\propto$ Eid des Hippokrates, zitiert nach Sass (Hrsg.), Medizin und Ethik, 1989 , S. 351 .

79 Den Konflikt veranschaulicht \$1 Abs. 2 Satz I der Berufsordnung fủr dic deutschen Ärzte von 1988 (Musterberufsordnung), DABBI. 85 (1988), 2199 fl.: $\bowtie$ Aufyabc des Arzt's ist ('s, das Leben zu erhalten, dic Gesundheit zu schutzen und wiederherzustellen sowie Leiden zu lindern.*

8o Es ist letzelich nur cine Frage der Terminologic, ob man auch hicr von einer Pflichtenkollision spricht (so Jescheck (Fn. 48 ), S. 328), oder $\$ 34$ StGB unmittelbar Anwendung finden laßt (Kuper, JuS r97r, 474 (475): Lenckner, in: Sch/Sch, vor $\$ 32$ ff., Rdnr. 71 ; Roxin, Strafrecht AT 1, 1992, \& 16, Rdnr. I02). Der Notstand setze jedoch keine Pflichtbeziehung zu einem der kollidierenden Guter yoraus, so daß an dem Bcgriff der Pflichtunkollısion festgehalten werden soll, da er genau die Situatuon beschreibt, in der sich der Ar\% befindet.

8r Jahnke (Fn. S1); Wusscls (Fn. 68); Eser, in: Auer/Menzel/Eser (Fn. 22), S. go, weist ihn zwar nicht grundsätzlich zurück, hàt thn aber für prohlematisch.

82 Merkel (Fn. 31), S. 93. 
nicht zulässig: »Entscheidend ist letztlich jedoch nicht, ob das durch die Tat geschützte Rechtsgut seinem absoluten Rang nach höherwertig, sondern ob es in der konkreten Lebenssituation schut\%würdiger ist. $\ll^{8_{3}}$ Die generelle Schutzwürdigkeit des Lebens hier zurücktreten zu lassen, muß erlaubt sein. Es kann nicht darauf ankommen, das verlöschende Leben um jeden Preis nicht verkürzen zu wollen, wenn eine intensive Schmerztherapie erforderlich ist. In Fällen der indirckten Sterbehilfe ist die Pflicht, nicht zu töten, zugunsten der Pflicht zu begrenzen, Schmerzen zu lindern. Der Arzt ist aufgrund cincr Pflichtenkollision gerechtfertigt. Das gilt auch dann (und gerade dann), wenn das Risiko der Lebensverkürzung zur Gewißheit wird. Erkennt man eine derartige Form der Schmerzlinderung grundsätzlich als straffrei an, was ja im Ergebnis einhellig getan wird, so stellt sich allerdings die Frage, wo die Grenze zur direkten Euthanasie liegt oder liegen kann.

\section{b) Die Grenze zur direkten Euthanasie}

Zur Abgrenzung wird die Zulässigkeit der indirekten Sterbehilfe unter einen Vorbehalt gestellt: die mögliche Lebensverkürzung darf nur unbeabsichtigte Nebenfolge sein. Ist der Tod aber Hauptziel und Mittel zum Zweck der Leidensbeendigung, geht die indirekte in die direkte Euthanasie über und ist nicht mehr straflos ${ }^{84}$. Dies ist unter Geltung des Prinzips der Doppelwirkung allgemein anerkannt und stellt einen Ausweg aus dem Dilemma der Pflichtcnkollision dar, nicht beiden Pflichten gleichzeitig nachkommen zu können ${ }^{8}$. Auf dicse Weise verschiebt sich allerdings die Entscheidung über die Strafbarkeit wieder in den subjektiven Bereich. Es besteht die Gefahr, daß Handlungsmotive die Grenze zwischen gerechtfertigtem und rechtswidrigem Handeln ziehen ${ }^{86}$, wenn allein die Unterscheidung zwischen gezielter und nicht gezielter Lebensverkürzung den Maßstab bildet ${ }^{87}$.

Zur direkten Euthanasie verbleibt in jedem Fall eine Grenze. In Fällen der Lebensverkürzung durch cine Schmerztherapie leitet sich die Einschränkung des Tötungsverbotes aus der erforderlichen Schmerzlinderung ab. Diese Begrenzung fordert, daß der Arzt nicht mehr tut, als Schmerzen zu bekämpfen ${ }^{3 \&}$. Der langfristige Erfolg einer solchen Behandlung stellt $z$ war eine tatbestandliche Tötung dar. Diese ist aber dann gerechtfertigt, wenn der Patient im Stadium einer unheilbaren Krankheit oder im Angesiche des nahenden Todes nur noch durch solche Dosierungen von Schmerzmitteln Leidenslinderung erlangen kann, die über kurz oder lang zu seinem frühzeitigen Tod führen. Damit wird ein wichtiger Unterschied deutlich. Die indirekte Sterbehilfe ist durch eine ärztliche Schmerzbehandlung eingerahmt und ausschließlich inncrhalb dieser zulässig. Bei der direkten Euthanasie hingegen fehlt es schon an der medizinisch indizierten Pflicht, Leiden mittels eincr unmittelbaren Tötung zu beenden.

\section{c) Stellenwert und medizinische Praxis}

Die Bedeutung der indirekten Sterbehilfe ist insgesamt zurückgegangen, da es inzwischen viele Methoden ohne großes Risiko gibt. Zudem besitzen Betäubungsmittel

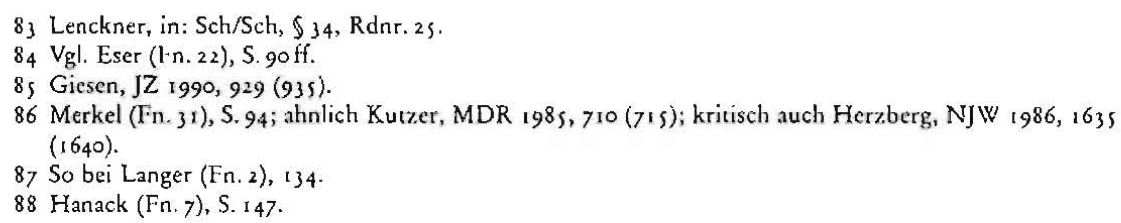


oft eine die Vitalität stabilisierende Kraft, wogegen Schmerzen häufig den Tod beschleunigen oder gar mitverursachen ${ }^{89}$. Gleichwohl ist die indirekte Euthanasie nicht gänzlich entbehrlich ${ }^{\circ}$. In der Praxis allerdings zeigr sich zumeist ein ganz anderes Bild der medizinischen Schmerztherapie. So werden starke schnerzstillende Mittel oft nur eingeschränkt oder gar überhaupt nicht in manchen deutschen Kliniken verwendet. Orales Morphin beispielsweise wird entgegen den Erfahrungen in anderen Ländern nur wenig benutzt ${ }^{91}$. Die Betäubungsmittcl-Verschrcibungsverordnung von I 974 hat zusammen mit der zu pauschalen Kriminalisierung der Verwendung von Opiaten durch das Betäubungsmittelgesetz zu einenı Rückgang der Vcrwendung von Morphinpräparaten geführt. Bezogen auf die Anzahl der Einwohner, ist der Verbrauch in Deutschland etwa zwanzigmal niedriger als in Dänemark und ctwa zehnmal geringer als in England ${ }^{9 x}$. Daneben tun die unangebrachte Angst vor Suchtanzeichen des Todkranken und die fragliche Grenze der Zulässigkeit der indirekten Sterbehilfe ihr übriges, um zu einer Gleichgültigkeit gegenüber dem Leidenden und zur Vernachlässigung der ärztlichen Pflicht beizutragen, Schmerzen zu bekämpfen. Solchen Zuständen entgegenzurvirken, muß auch Aufgabe des Rechts sein. Danit licße sich nicht zuletzt die Problematik der direkten Euthanasie entschärfen. Denn allzu oft hat die Forderung nach deren Freigabc ihren Ursprung in der Angst vor nicht ausreichender Schmerzbekämpfung93.

\section{Direkte Euthanasie}

Wird das Leben eines Menschen gezielt und unmittelbar beendet, um ihm Qualen zu ersparen, spricht man von aktiver oder direkter Euthanasie ${ }^{94}$. Der Tod ist nun nicht mehr unbeabsichtigtc Nebenfolge, sondern Mittel zum Zweck der Leidensbeendigung. Es geht nicht um cine mittelbare Verkürzung, sondern um die unmittelbare Beendigung des Lebens. Sterbehilfe beschränkt sich auf die Hilfe zum Stcrben und ist als Tötungsdelikt nach deutschem Recht strafbar. Die Tötung auf Verlangen ist nach $\$ 216 \mathrm{StGB}$ mit Haftstrafe von sechs Monaten bis zu fünf Jahren bedroht. Statistisch spielt diese Vorschrift jedoch kaum eine Rolle. Die Verurteilungsrate bewegt sich scit 1970 jährlich im einstelligen Bereich"s, wobei offen bleiben muß, ob es sich hierbei immer um Fälle der direkten Euthanasic handelt.

Im Zuge der Fälle Hackethal ${ }^{96}$ und Wittig97 ist in Deutschland auch die aktive direkte Euthanasic Gegenstand verhaltener, öffentlicher Diskussionen geworden. Dancben haben die teilweise gewaltsam verhinderten Auftritte des australischen Philosophen Peter Singer im Jahr 1989 die Aufmerksamkeit der Öffentlichkeit auf diesen Problombereich gclenkt $t^{98}$. Hierbei ist zu bcobachten, daß die Euthanasiedebatte im wesentlichen eine Singer-Debatte ist. Mit Blick auf das "Euthanasieprogramm « der

\footnotetext{
89 Schaefer, Medizinische Ethik, 1983 , S. 2 rof.; vgl. auch v. Lutterotti, in: Eser (Hrsg.) (Fn. 2), S. 294.

$90 \mathrm{Vgl}$. Mayrhofer/Porges, in: Eser (Hrsg.) (Fn. 2). S. 121 ; siehe auch die Einzelbegrundung zu $\$ 214 \mathrm{a}$ Alternativentwurf Sterbehilfe, 3., S. 23 .

9r Uhlenbruck, ZRP 1986, 209 (216); siehe auch Twycross, in: Fser (Hrsg.) (Fn. 2), S. 1 is.

92 Hassenstein, Nicht zum Aushalten, DIE ZEIT Nr. 2 vom 7. Januar 1994, S. 33.

93 Hanack (Fn. 7), S. 134; Uhlenbruck (Fn.91), $216 \mathrm{f}$.

94 Vgl. Ehrhardt (Fn. 6), S. 6; Engisch, in: Eser (Hrsg.) (Fn. 2), S. 318; Hanack (Fn. 7), S. 145; Jähnke, in: LK, vor $\$ 211$, Rdnr. 12; Schreiber (Fn. 7), 339; Sporken, in: Eser (Hrsg.) (Fn. 2), S. 277.

95 Koch (Fn. 3), S. 53

96 Dazu Herzberg, NJW 1986, 1635.

97 Siehe oben Fn. is.

$98 \mathrm{Vgl}$. Merkel, Der Streit um Leben und Tod, DIE ZEIT Nr. 26 vom 23 . Junt 1989, S. 13 ff.
} 
Nationalsozialisten scheut man es, sich mit direkter Sterbehilfe zu beschäfrigen und beschränkt sich statt dessen oftmals auf eine "Diskussion über die Diskussion «. Seit längerer Zeit ist auch in der deutschen Strafrechtswissenschaft die direktc Euthanasie Gegenstand juristischer Betrachtungen. In erster Linie geht es aber darum, wie Einzelfälle de lege lata am ehesten in einer für den Täter gerechten Weisc gelöst werden können'99.

Die in der Literatur bereits vorhandenen Regclungsansätze zur dirckten Euthanasie offenbaren ein breites Spektrum an Meinungen und damit auch ein gewisses $\mathrm{Maß}$ an Unsicherheit ${ }^{100}$. So soll es in jedem Fall uneingeschränkt bei der Strafbarkeit bleiben $^{101}$. Dagegen finden sich Stimmen, welche die Rechtswidrigkeit aufgrund einer Pflichtenkollision oder eines Notstandes nach $\$ 34$ StGB verneinen ${ }^{102}$. Auch wird in besonderen Fällen die Möglichkeit der Entschuldigung analog $\mathbb{S} 35 \mathrm{StGB}^{103}$ oder ein Abschen von Strafe in Anlehnung an $₫ 60 \mathrm{StGB}^{104}$ erwogen. De lege ferenda werden für $\$ 2$ I 6 StGB verschiedene Vorschläge zur Neufassung gemacht ${ }^{10 s}$. Die Vorschrift soll durch besondere Rechtfertigungsgründe ${ }^{166}$ oder Strafverzichtsmöglichkeiten ${ }^{107}$ ergänzt werden. Schließlich setzt man sich dafür ein, die Vorschrift ganz zu streichen und damit der Einwilligung auch im Bereich der Tötungsdelikte rechtfertigendes Gewicht zu geben ${ }^{10 \%}$.

\section{a) Rechtfertigung?}

Dem Arzt oder einem Dritten dic Möglichkeit einer Rechtfertigung zu eröffnen, hat sicherlich einen Vorteil. Jede aktive Lebensverkürzung ließe sich unabhängig von "indirekten « oder "direkten « Absichten nach einheitlichen Gesichtspunkten beurteilen ${ }^{109}$. Es entfiele auch der Zwang, bei der passiven Euthanasie den technischen Behandlungsabbruch als Unterlassen einordnen zu müssen, um so die Garantenpflicht verneinen zu können. Aus der Notwendigkeit, im Rahmen einer ärztlichen Schmerztherapie den Mediziner auch dann zu rechtfertigen, wenn das Leben des Patienten mit Sicherheit verkürzt wird, folgt jedoch noch nicht, die Tötung auf Ver-

99 Oft wird ein Fall angefuhrt, der sich angeblich in Schweden zugetragen haben soll. Nach einem Unfall erschlägt der Beifahrer den eingekJemmten Fahrer eines LKW auf dessen Flehen hin mit einer Axt, um ihm den Verbrennungstod zu ersparen; vgl. Otto (Fn. 12), S. 60; Simson, in: Escr (Hrsg.) (Fn. 2), S. 324 f. sowic Alternativentwurf Sterbehilfe, S. 35 .

rco Hocrster beispiclsweise hat von 1986 bis 1990 allein vier unterschiedliche Vorschlige zur Ergänzung des $\$ 216$ StGB gemacht; siehe Koch (lin. 3), S. is 8 f.

101 Jähnke, in: L.K, Rdnr. 14; Langtr (Fn. 2), 135; Trondle, ZStW 99 (i987), 2s (41 f.); Wessels (Fn. 68), S. 6.

102 Herz.berg (Fn. 96), 1639 ff.; Merkcl, in: Hegselmann/Merkel (Hrsg.) (Fn. 31), 97; Otto (Fn. 12), S. 99 f.; Schrciber (Fn. 7), 339; Simson, in: Evers/Friauf/Hanack/Reinhardt (Hrsg.), Festschrift für Schwinge, 1973 , S. 110.

103 V. Dellinghausen (Fn. 22), S. $337 \mathrm{ff}$; Hirsch, in: Küper (Hrsg.) (Fn. 48), S.615; Mollering (Fn. 22), S. 47 f.

104 Hanack (Fn. 7), S. iss f; Arthur Kaufmann (Fn. 40); Roxin, in Blaha/Gutjahr-Loser/Nicbler (Hrsg.), Schutz des Lebens - Reche auf Tod, 1978, s. 93 f.; vgl. auch Oto (Fn. 12), S.6I.

105 Eine Zusammenstellung findet sich bei Koch (Fn. 3), S. 158 f.

106 Die Humanistische Union z. B. will dic Rechtswidrigkeit dann entfallen lassen, wenn vdie Tat begangen wird, um einen menschenwurdigen Tod herbeizuluhren $x$; Deutscher Bundestag, Stenographisches Prorokoil zur offentlichen Anhorung zum Thema "Sterbchilfex des Rechtsausschusses, 10. Wahlperiode, 51. Sitzung, is. Mai 1985, Anlage S. 145; ahnlich Klug, Alternativentwurf Sterbehilfe, S. 36; weitgehend auch Hoerster, NJW 1986, 1786 (1790, 1792); ders., in: Sass (Hrsg.) (Fn. 78), S. 295.

107 "Das Gericht kann unter den Voraussetzungen des Abs. I von Strafe absehen, wenn die Tötung der Beendigung eines schwersten, vom Betroffenen nucht mehr zu ertragenden Leidenszustandes dient, der nicht durch andere $\mathrm{Ma} ß n$ nahmen behoben oder gelindert werden kanna, $\$ 216 \mathrm{Abs}$. 2 Alternativentwurf Sterbehilfe. Abs. i soll unverändert bleiben.

108 So vor allem Arthur Kaufmann (Fn. 40), 124 und Rudolf Schmitt, JZ 1985, 365 (366); ders., JZ 1979, 462 (467): "(nahezu) tote Vorschrif(*.

109 Merkel, in: Hegselmam/Merkel (Hrsg.) (Fn. 31), S. 97 
langen nach denselben Gesichtspunkten rechtfertigen zu können. Dabei ist der phänomenologische Unterschied zur indirekten Euthanasie zu berücksichtigen. Es geht nicht darum, cine Schmerzbekämpfung mit lebensverkürzender Wirkung zu rechtfertigen, sondern die Tötung des Leidenden selbst als Mittel zur Schmerzbefreiung. Die Situation des Arztes ist nicht mit derjenigen bei der indirekten Euthanasie vergleichbar. Der Pflicht, lebensverkürzende Maßnahmen zu unterlassen, steht keine Handlungspflicht gegenüber. Die Tötung als Mittel zum Zweck der Schmerzbeendigung ist nicht vom ärztlichen Auftrag gedeckt ${ }^{100}$. Stellt sich der Gnadentod als ultima ratio dar, kann der Arzt folglich nicht aufgrund einer Ptlichtenkollision, sondern allenfalls unmittelbar wegen Notstands nach $\S_{34} \mathrm{StGB}$ gerechtfertigt werden, sofern das Interesse am verbleibenden Leben als nachrangig gegenüber dem Interesse an der Beendigung der Leiden crachtet wird.

Abgesehen von der Frage, ob eine solchc H-landlung ein angemessenes Mittel nach $\$ 34$ Satz 2 StGB sein kann" "', ist dieser Weg nicht mit der Funktion des $\$ 216$ StGB zu vereinbaren. Der Entwurf von 1962 läßt ausdrücklich erkennen, daß die Fälle der aktiv-dirckten Euthanasie zum »wesentlichen Anwendungsbereich « der inhaltlich unveränderten Tötung auf Verlangen gehören sollten"'2. Eine Möglichkeit der Rechtfertigung höbe nun die Entscheidung des Gesetzgebers wieder auf, die direkte Euthanasie gerade nicht zuzulassen" "3. Das "Recht auf Sterben * müßte um ein "Recht auf Tötung « ergänzt werden. Eine Rechtfertigung relativierte den Stellenwert des Rechtsgutes Leben in der gesamten Rechtsordnung und wäre eine zu große Einschränkung des strafrechtlichen Lebensschutzes. Einen solch wcitreichenden, rechtspolitischen Schritt zu gehen, ist allenfalls de lege ferenda möglich und kann nicht das Ergebnis einer Strafrechtsauslegung sein. Die objektive Kategorie ${ }^{2}$ Rechtswidrigkeit « ist nicht der geeignete Bereich, un als nicht strafwürdig empfundene Einzelfälle lösen zu können.

Rechtspolitisch mag es durchaus vertretbar sein, dem Arzt die Möglichkeit einer Rechtfertigung zuzubilligen. Es müßte aber in jedem Fall einer zu pauschalen Abwägung mittels konkreter Kriterien und einer ausgefeilten Kasuistik begegnet werden, um Mißbrauch und Ausuferung zu verhindern ${ }^{14}$. Dafür fehlen aber derzeit jegliche Ansatzpunkte, die auch dic Vorschläge zur Ergänzung des $\ 2$ I 6 StGBirs nicht liefern können, welche dic Rechtswidrigkeit unter Verwendung von Formulierungen wie "qualvolles Leiden", "menschenwürdiger Tod" oder "lebenswertes Leben "16 entfallen lassen wollen. Dies gilt erst recht für den Vorschlag, die Vorschrift ganz zu streichen und die Tötung auf Verlangen nur durch eine Sittenwidrigkeitsklauscl ähnlich der des $\ 226$ a StGB einzuschränken' ${ }^{17}$. Ein solcher Ansatz ist schon allein deshalb fraglich, da der Getötete einer behaupteten Einwilligung nicht mehr widersprechen kann, und die Umkehr der Beweislast nicht mit dem Grundsatz in dubio pro reo vereinbar ist. Man verlagerte zudem das Problemfeld der Euthanasie aus dem ärztlichen Bereich in den alltäglichen und öffnete durch den auslegungsfä-

$10 \mathrm{Vgl}$. Resolution der Deutschen Gesellschaft für Chirurgie vom 10. 4. 1979, IV., abgedruckt in: Altern.tiventwurf Sterbehilfe, S. 44 ff.; Entschließung des 84. (1981) und 87. Deutschen Ärztetages (1984), DÄBl. $78\left(\mathrm{rg} \mathrm{SI}_{\mathrm{I}}\right), \mathrm{I} 88$ und DÄBl. $81(1984), 1764$.

Auch in den Niederlanden geht man nicht von wnormalen $*$ arzctichen $\mathrm{Ma}$ ßnahmen aus, vgl. van der Wal, Biocthics Volume 7, Number 4, 1993, 330 (334).

I1, Die Situation des Arztes als Notstandslage qualıfizieren auch Geilen (Fn. 10), S. 26f. und Schreiber (Fn. 7), 339, verneinen jedoch die Angemessenheit.

112 Verhandlungen des Deutschen Bundestages, Drucksache IV/650, S. 275.

113 Roxin, in: Wolter (Hrsg.), 140 Jahre Goltdammer's Archiv fur Strafrecht, 1993, S. $187 \mathrm{f}$

14 So auch Merkel, in: Hegselmann/Merkel (Hrsg.) (Fo. 31), S. 97.

ins Eine Übersicht bei Koch (Fn. 3), S. is $8 \mathrm{f}$.

116 Ein objektiv slebensunwertes Leben gibt es nicht; siche Durıg, in: Maunz/Durig/Herzog, GG, 8. Auflage, 30. Lieferung (Stand September 1993), Art. 2 II, Rdnr. 10. 
higen und auslegungsbedürftigen Rechtsbegriff der "guten Sitten « Mißbrauch Tür und Tor. Eine Regelung kann nicht auf die gewünschten Fälle begrenzt werden, wenn die Grenzen dafür fehlen.

\section{b) Entschuldigung?}

Kommt de lege lata eine objektive Rechtfertigung aufgrund der generellen Ausstrahlungswirkung eines solchen Schrittes keinesfalls in Betracht, bietet sich die Möglichkeit der subjektiven Entschuldigung in konkreten Ausnahmcfällen an. Für Pcrsonen, die dem Getöteten in einer Weise nahestehen, daß sie wegen dessen Lage unter einer besonderen psychischen Drucksituation stehen, kann der entschuldigendc Notstand nach $\$ 3$ S StGB unmittelbar Anwendung finden ${ }^{\text {rts }}$. Dagegen spricht nicht schon die Tatsache, daß eine Person Inhaberin des verletzten wie auch geschürzten Rechtsguts ist ${ }^{\prime 19}$. Zwar sind Leben und körperliches Wohlbefinden nicht glcichwertig, jedoch nur einer Person zugeordnet, so daß sich von diesem Erfordernis aufgrund der bei der indirekten Sterbehilfe angeführten Überlegungen zur rechtfertigenden Pflichtenkollision eine Ausnahme machen ließc. Diescr problematische und fragliche Vorbehalt ist jedoch nicht zwingend erforderlich. Der von der Rechtsprechung bisher noch nicht anerkannte, übcrgesctzliche entschuldigende Notstand, vielfach auch entschuldigende Pflichtenkollision genannt, ist in Extremfällen der dirckten Euthanasie und nur um diese kann cs de lcge lata gehen - anwendbar ${ }^{120}$. Das gilt auch für den Arzt, dem der unmittelbare Rückgriff auf $\$ 35 \mathrm{StGB}$ verwehrt ist, da ihm das vorausgesetzte nahe Verhältnis in der Regel fehlen wird.

Der Grund für die Entschuldigung ergibt sich nun weniger aus dem Gedanken der Unzumutbarkeit, als aus dem Zusammentreffen zweier Gesichtspunkte"2t. Erstens ist die Tat insgesamt $z$ war rechtswidrig, weil die Rechtfertigung einer Tötung auf Verlangen aus den dargestellten, grundsätzlichen Erwägungen nicht erfolgen kann. Das Unrecht wird jedoch in dem Maße gemildert, als dem erhaltenen Rechtsgut bzw. der nachgekommenen Pflicht im konkreten Fall rechtliche Bedeutung zukommt. Die Minderung des Unrechts verringert wiederum das von dessen Umfang abhängige Schuldmaß entsprechend. Zweitens kommt schuldmindernd hinzu, daß der Täter einem Motivationsdruck des Gewissens ausgesetzt war, der ihn zum Handeln bewog, weil cr auch in dem Fall schwere sittliche Schuld auf sich nähme, wenn er nicht handelte ${ }^{122}$. Um die analoge Anwendung des $\$ 35 \mathrm{StGB}$ zu rechtfertigen, muß die psychische $Z$ wangslage der vom entschuldigenden Notstand verlangten Stärke entsprechen, ohne daß dabei eine besondere persönliche Nähe zum Getöteten notwendig wäre. Eine Entschuldigung kann nur dann in Frage kommen, sofern sich die Tötung auf Verlangen als ultima ratio darstellt, ihr Unrechtsgehalt wesentlich gemindert ist und der Täter in schwerer Gewissensnot gehandelt hat ${ }^{123}$. Gleichwohl wird dies für den Mediziner nur äußcrst selten gelten können, da ihm eine solche Handlung berufsethisch grundsätzlich verboten ist.

Dieser Ansatz läßt das Frcmdtötungsverbot einerseits unangetastet, bietet aber andererseits die Möglichkeit, im konkreten Einzelfall eine gerechte Lösung zu finden und den Tätcr zu entschuldigen, dessen Tat einerseits objektiv von der Rechtsord-

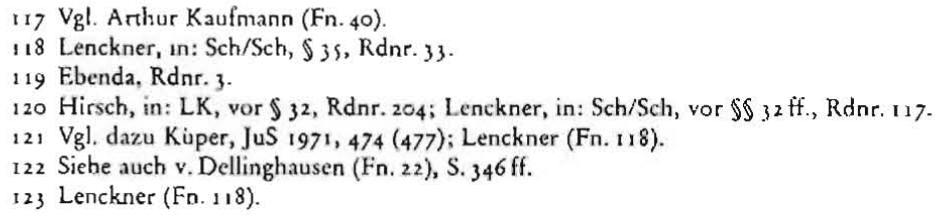


nung nicht akzeptiert werden kann, sein Handeln ihm andercrseits im Zusammenspiel beider eben genannten Aspekte subjektiv nicht vorwerfbar ist.

\section{c) Strafverzicht?}

Erscheint der Weg der Entschuldigung als nicht gangbar, verbleibt noch die Frage, ob unter Umständen auf eine Bestrafung verzichtet werden kann. Materiellrechtlich käme allentalls einc Anwcndung des $\$ 60$ StGB in Betracht, wenn die Folgen der Tat den Täter so schwer getroffen haben, daß die Verhängung einer Strafe verfehlt wärc. Dies ließe sich bejahen, wenn die Tötung auf Verlangen den Verlust eines Angehörigen zur Folge hat und dadurch kein Strafbedürfnis mehr besteht ${ }^{224}$. In Verbindung mit $\ 153$ b StPO könnte so bereits die Staatsanwaltschaft davon absehen, öffentlich Klage zu erheben. Sofern $\ 216$ StGB als selbständiger 'Tatbestand interpretiert wird, bietet $\$$ I 53 Abs. I StPO die Möglichkeit, auch in diesen Fällen nach dem Opportunitätsprinzip bei geringer Schuld und fehlendem öffentlichen Interesse auf eine Strafverfolgung zu verzichten. Allerdings wird angesichts der Bedeutung des strafrechtlichen Lebensschutzes und der gegensätzlichen Euthanasiediskussion ein solches Interesse kaum verneint werden können.

Die Verfasser des Alternativentwurfes Sterbehilfe wollen dem Gericht die Möglichkeit eröffnen, ohne das Erfordernis schwerer Tatfolgen für den Täter nach Schuldspruch und Urteil von der Strafe abzusehen. "Es geht allein darum, daß in einer extremen, für Täter und Getöteten gleichermaßen existenziellen Situation die Entscheidung des Täters, aus mitmenschlichen Gründen die Achtung vor fremden Menschenleben gegenüber der ausweglosen Not des Leidenden zurücktreten zu lassen..., unter bestimmten Voraussetzungen ohne strafrechtliche Sanktionen bleiben kann. «"2s Dieser Ansatz entspricht etwa den oben bei der Frage der Schuld angestellten Übcrlegungen. Er gcht jedoch insofern über diese hinaus, als er einerseits muneingeschränkt die Antastbarkeit als ultima ratio normiert. Man möchte von der Strafbarkeit der direkten Sterbehilfe grundsätzlich eng begrenzte Ausnahmen machen können. Die enge Begrenzung wird jedoch bereits durch die Tatsache relativiert, daß dem Gericht ein Beurteilungs- und Entscheidungsermessen zukommt, welches nicht weiter eingerahmt wird. Eine derartige Regelung sorgte für mehr Unsicherheit und verwischte eher die Grenzen zwischen strafbarer und straffreier Tötung auf Verlangen, als daß sie solche zöge. Die Regelung des Alternativentwurfes ist vicl zu unscharf, als daß sie den Vorstellungen der Verfasser über Art und Umfang straffreier direkter Euthanasie gerecht werden kann, was im übrigen auch für die Vorschläge zur passiven und indirekten Sterbchilfe gilt. Zu weit auslegbare Tatbestandsmerkmale und cin nicht näher begrenzter Spielraum der Gerichte lassen die Möglichkcit, in dicser Weise einen gerechten rechtlichen Weg zu finden, fraglich werden. Wenn es nimmer wieder einmal erbetene Tötungen geben wird, bei denen die Unentrinnbarkeit der Situation und die Tragik des Geschehens jeden menschlichen Vorwurf verstummen läßt $\varkappa^{126}$, ist es widersprüchlich, dem Täter seine Handlung dennoch vorzuwerfen und nur von einer Bestrafung abzusehen. Es könnte letztendlich der Eindruck entstehen, daß man dem Täter einer Tat, die ihm niemand sittlich zum Vorwurf zu machen wagt, auch rechtlich an einer Bestrafung vorbeihelfen will ${ }^{127}$

\footnotetext{
124 Hirsch, in: LK, I r. Auflage 1992. $\$ 60$, Rdnr. 23.

125 Siehe Alternativentwurf Sterbehilfe, Begrundung zu $\$ 216,1.4 .$, S. 36 .

I 26 Roxin, in: Blaha/Gutjahr-Loser/Nicbler (Hrsg.) (Fn. 104), S. 93 f.

127 So zutrelfend Herzberg, NJW 1986, r635 (1643); ahnlich v. Dellinghausen (Fn. 22), S. 349 f., 489 f.
} 


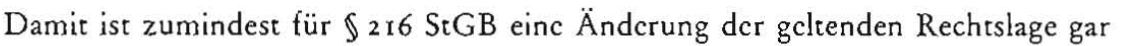
nicht erforderlich. Wenn es Gründe gibt, die an der Vorwerfbarkeit einer Törung auf Verlangen Zweifel lassen, so ist dies cine Frage der Schuld und nicht des Strafverzichts unter Schuldspruch. Die bestehenden Möglichkeiten der Strafrechtsauslegung reichen aus, um extreme Einzelfälle lösen zu können. Soll darüber hinaus die aktivdirekte Euthanasie prinzipiell zugelassen werden, so bedarf es zunächst grundsätzlicher juristischer wie ethischer Überlegungen. Eine Rechtfertigung ist nur möglich, und dann auch erforderlich und konsequent, wenn man sich unter Abwägung der Folgen für Recht und Gesellschaft für diescs Prinzip cntschieden hat.

\section{Das niederländische Modell}

Die Niederlande sind bisher das einzige europäische Land, welches sich für ein solches Prinzip entschieden hat. Dort wird schon seit den sicbziger Jahren öffentlich auch über die Zulässigkeit einer aktiv-direkten Sterbehilfe diskutiert. Man orientiert sich hierbei jedoch an einer anderen Terminologie. Die Einteilung in »aktive» und "passive« Formen wird oft mit der Begründung abgelehnt, man könne in diesem Bercich Tun und Unterlassen gar nicht trennen ${ }^{128}$. Die Abgrenzung ist aber wie beim deutschen Recht von Bedcutung. Der gesamte Problembereich wird umschrieben mit medizinischen Entscheidungen, die das Ende des Lebens betreffen (medical decisions concerning the end of life, MDEL) ${ }^{129}$. Die in Deutschland der passiven und indirekten Sterbehilfe zugeordneten Verhaltensweisen sind nur vapparent forms ${ }^{\prime 30}$ der Euthanasie, die üblicherweise nur die ausdrücklich und crnsthaft erbetene aktivdirekte Sterbehilfe umfaßt. Daneben wird gewöhnlich kein Unterschied in der Bewertung von Euthanasic und Bcihilfe zur Selbsttötung gemacht. Gegenstand der derzeirigen Diskussion ist die Frage, ob b̌w. inwieweit lebensbeendende Maßnahmen ohne einen ausdrücklichen und gültigen Willen des Betroffenen (life-termination acts without the explicit request of the patient, $L A W E R$ ) zulässig sein können'31.

\section{a) Bisherige Rechtslage}

Besondere Strafvorschriften zur Sterbehilfe gibt es nicht, obwohl man sich schon seit 1970 un eine Reform des Strafgesetzbuchs bemüht. Nach Art. 293 niStGB ist die Tötung auf Verlangen mit bis zu zwölf Jahren und nach Art. 294 nlStGB die Beihilfe zum Selbstmord mit bis zu drei Jahren Haft bedroht. Seit Anfang der achtziger Jahre haben die Gerichte in Anlehnung an die Auffassungen der Ârzteschaft Kriterien entwickelt, nach denen Fälle der direkten Sterbehilfe und der Beihilfe zur Selbsttötung zulässig sein können. Inzwischen ist man sich einig, wann auch nach bisherigem Recht cine Straffrciheit in Betracht kommt. Die Möglichkeit einer Rechtfertigung wird jedoch nur Ärzten zugestanden, wobei in der Regel auf Gesichtspunkte des Notstands oder der Übermacht kollidierender Pflichten (force majeure) zurückgegriffen wird.

I 28 Vgl. Leenen (Fn. 4), S. 4

I 29 So van der Maas/van Delden/Pijnenborg/Looman, The Lancet 1991, 669.

r 30 Van der Wal (Fn. 114), 332.

13i Dazu van der Wal (Fn. 114), 330 ff. 
Mittlerweile werden Fälle der direkten Euthanasie und der Beihilfe zum Suizid strafrechtlich nicht mehr geahndet ${ }^{132}$, sofern die aufgcstcllten Voraussetzungen vorliegen. Diese wurden 1984 von der Königlich-Niederländischen Gesellschaft zur Förderung der Heilkunde (KNMG) veröffentlicht' ${ }^{13}$. Der Betreffende muß seincn Tod ausdrücklich gewünscht haben (a), über seine Lage wohlinformiert und sich ihrer bewußt sein (b), den Todeswunsch dauerhaft äußern (c), sein Leiden muß unaushaltsam und sein Zustand ohne Aussicht auf Besserung sein (d). Schließlich hat der ausführende Arzt mindestens einen Kollegen vorher hinzuzuziehen (e). I 990 entwickeltc das Justizministerium in Zusammenarbcit mit der KNMG ein Meldeverfahren zur direkten Euthanasie und Beihilfe zum Selbstmord. Der ausführende Arzt stellt in diesen Fällen keinen Totenschein über einen natürlichen Tod aus, sondern unterrichtet den örtlichen Leichenbeschauer schriftlich. Dieser meldet die Sache der zuständigen Staatsanwaltschaft, die nach dem in den Niederlanden allein geltenden Opportunitätsprinzip von einer Strafverfolgung in der Regel absieht, sofern obige Kritcrien erfüllt sind.

\section{b) Empirische Untersuchungen}

Um sich über Umfang und Bedeutung der Euthanasiepraxis in den Niederlanden Klarheit zu verschaffen, beauftragte die holländische Regierung 1990 eine Kommission mit der Untersuchung dieser Frage. Den Vorsitz führte Prof. Remmelink, Generalstaatsanwalt des niederländischen Obersten Gerichtshofes. Es ist eine landesweite Studie erstellt worden, deren erste Ergebnisse im Scptember 199 I veröffentlicht wurden ${ }^{134}$. Man führte dafür zwei verschiedene Befragungen bei 405 Medizinern aus unterschiedlichen Disziplinen durch und untersuchte 7000 von insgesamt 4 I 587 Todesfällen, die sich im Zeitraum von August bis Dezember 1990 ercigneten. Der Anteil der MDEL lag bei $38 \%$, wovon jeweils $17,5 \%$ auf die passive (nontreatment decisions) und die indirekte Sterbehilfe (allevation of pain and symptoms) fielen. Der direkten Sterbehilfe kam ein Prozentsatz von eta r, 8 und der Beibilfe zum Selbstmord einer von $0,3 \mathrm{zu}$. Schlicßlich verzeichneten die $L A W E R$ einen Anteil von $0,8 \%$ der Todesfälle ${ }^{13 s}$

Nach dieser und ciner anderen Studie ${ }^{136}$ wird aktiv-direkte Euthanasic zusammen mit Beihilfe zum Selbstmord in den Niederlanden in etwa 2000 Fällen pro Jahr crteilt. Dabei verlangen jährlich 25000 Menschen von ihren Ärzten, sie mögen ihnen zur Seite stehen, wenn ihr Leiden unerträglich werde. 9000 davon äußern den ausdrücklichen und ernstlichen Wunsch zu sterben, wovon letztlich weniger als ein Drittel angenommen wird. Oft können andere Möglichkeiten der Leidensminderung gefunden werden. Bei Menschen im Alter über so Jahren wird direkre Euthanasie oder Beihilfe zur Selbsttötung häufiger durchgeführt als bei jüngeren Personen, wobei jenseits der 80 Jahre dieser Anteil ab und der der non-treatment decisions zunimmt. Die mit Abstand häufigste (68\%) jenen Maßnahmen zugrunde liegende Krankheit ist Krebs. In 187 näher untersuchten Fällen wurde fast immer der Sterbewunsch wiederholt und ohne Druck vom Patienten gcäußert, welcher seine Lagc selbst cinschätzen konnte. Als Gründe für den Sterbewunsch wurden Verlust der

\footnotetext{
1321988 wurden beispielsweise 181 Fälle eingesteilt und nur 2 uberhaupt weiter verfolgt; vgl. Scholten, in: Eser/Koch (Hrsg.) (Fn. 3), S. 462.

133 Medisch Contact 1984, Nr. 31, 990 ff.; siehe auch Royal Dutch Medical Association (Fn. 4), s. 3.

134 Siehe zum folgenden van der Maas/van Delden/Pijnenborg/Looman (Fn. 129), 669ff.

135 Dazu Pijnenborg/van der Maas/van Delden/Looman, The Lancet r $993,1196 \mathrm{ff}$.

136 Diese befaßt sich ausschließlich mıt Hausärıten; vgl. van der Wal/van Eijk/Leenen/Spreeuwenberg (Fn. 4), I30 ff.; $135 \mathrm{ff}$.
} 
Würde ( $5 \%$ ), Schmerz (46\%), unwürdiges Sterben ( $46 \%$ ), Abhängigkeit von anderen $(33 \%)$ und Lebensmüdigkeit (23\%) genannt. Auffallend ist, daß in nur 10 der I 87 Fälle der Schmerz die alleinige Ursache für den Sterbewunsch war.

Die befragten Ärzte ließen eine hohe Bereitschaft erkennen, direkte Euthanasie oder Beihilfe zum Selbstmord zu leisten. $54 \%$ gaben an, diese Maßnahmen schon einmal getroffen zu haben, und $34 \%$ wären dazu unter bestimmten Umständen bereit. Lediglich I $2 \%$ ständen dafür nicht zur Verfügung, wovon jedoch $8 \%$ den Betreffenden an andere Ärzte weiter verwiesen. Am häufigsten haben diese Maßnahmen Hausärzte durchgeführt, am wenigsten dagegen Mediziner aus Pflegeheimen. Damit sieht eine große Mehrheit der holländischen Ärztc direkte Euthanasie und Beihilfe zur Selbsttötung als annehmbare Bestandteile der medizinischen Praxis an.

\section{c) Kammersache 22572}

Im April 1992 verfaßte die niedcrländische Regicrung aufgrund der Untersuchungsergebnisse einen Gesetzentwurf, der als Kammersache $22572^{137}$ im April 1992 der Zweiten Kammer des Parlamentes vorgelegt und von dieser am 9. Februar 1993 angenommen wurde. Der Vorschlag läßt die Vorschriften des Strafgeserzbuches unverändert, so daß die direkte Euthanasie auch weiterhin grundsätzlich strafbar bleibt. Der Entwurf ist eine Ergänzung des Gesetzes über die Leichenbestattung von 1990 und verankert das oben beschriebene, bercits bestehende Meldeverfahren gesetzlich. Um der Entwicklung in der Rechtsprechung nicht vorzugreifen, werden keine Voraussetzungen festgelegt, nach denen von einer Strafverfolgung abgesehen werden kann. Aufgeführt ist lediglich ein Fragenkatalog zur "ärztlichen Sorgfaltspflicht « in diesen Fällen, den der ausführende Arzt beantworten muß, wenn er den Leichenbeschauer informiert. Allerdings enthält die Kammersache 22572 einen Punkt, der teilweise heftig kritisiert wurde, da dafür noch keinerlei Kriterien entwickelt worden $\operatorname{sind}^{138}$. Dic Anwendbarkeit des Meldeverfahrens wird auch auf die Fälle erweitert, in denen kein ausdrücklicher Sterbewunsch vorgelegen hat. Kammersache 22572 ist am 30. November 1993 mit nur drei Stimmen Mehrheit auch durch die Erste Kammer (Senat) angenommen worden'19, so daß das Meldeverfahren nun den Status eines formellen Gesetzes erhalten hat.

\section{Abschließende Betrachtung}

Für sich betrachtet mag der Weg der Niederlande als pragmatisch, fortschrittlich, ja "human « erscheinen. Im Zusammenspiel mit anderen Entwicklungen an der Grenze von Leben und Tod offenbart sich jedoch deren eigentliche Bcdeutung. Der Lebensanfang ist bereits vcrfügbar geworden, das Lebensende wäre "der nächste Schritt $\alpha^{1} 4^{\circ}$. Direkte Euthanasie ist keine medizinische Notwendigkeit. Der "Schöne, neue Tod « ist vielmehr ein weiterer Baustein zu einer eben solchen Welt, in der alles möglich und nichts unmöglich ist. Der vielbeschworene "Dammbruch» bei der Bedeutung

137 Vgl. dazu Gevers, Journal of medical ethics 1992, Nr. I8, I 38 ff., sowie Royal Dutch Medical Assocration (Fn. 4), S. $3 \mathrm{ff}$.

138 Daneben wird fur unbefriedigend empfunden, daß der Arzt grundsatzlich strafbar und der Strafverfolgung ausgeliefert bleibt, auch wenn er sich an alle Kriterien halt; vgl. KNMG, Medisch Contact 1991 , Nr. 46, 1541 ff.; sowic Gevers (Fn. 157), 140.

$139 \mathrm{Vgl}$. SZ Nr. 278 vom 2. Dezember 1993, 48. Woche, S. 8.

140 Dazu Engisch, in: Grünwald/Miehe/Rudolphi/Schresber (Hrsg.), Festschrift für Schaffstein, 1975, S. Iff. 
des menschlichen Lebens hat in Wirklichkeit schon längst begonnen und scheint unaufhaltsam fortzuschreiten. Krankheit, Behinderung, Alter und Tod werden zu beherrschbaren und besiegbaren Risikofaktoren erklärt, deren Vermeidung jeglichen technischen oder genetischen Eingriff rechtfertigt. "Leid « und »Leben « bilden nicht mehr eine Einheit, sondern werden zu Gegensätzen.

Von der eingangs erwähnten sozialen Umbruchssituation lassen sich Parallelen zu früheren Entwicklungen ziehen'"1. Sowohl Utilitarismus wie auch Sozialdarwinismus basieren auf einem Denken, das der Zweckrationalität höchste Priorität einräumt. Lediglich die Perspektive unterscheidet sich. Doch dicse läßt sich leicht verschieben. Denn die Interessen des Einzelnen sind nicht von denen der Gesellschaft unabhängig, sondern von diesen geprägt. In einer Zeit, in der materielle Gesichtspunkte in immer stärkerem Maße Problemlösungen beeinflussen, ist es unverantwortlich, säkularisierte, christliche Wertvorstellungen wie dic Unverfügbarkeit des menschlichen Lebens aufzugeben. Wann Kosten-Nutzen-Erwägungen in einer leistungsorientierten und leistungsabhängigen, sich selbst immer weiter rationalisierenden Gesellschaft über »lebenswertes" und „lebensunwertes Leben entscheiden, ist dann nur eine Frage der Zeit ${ }^{142}$. Auch das holländische Modell bietet dagegen keinen wirksamen Schutz, da es bewußt der weiteren Entwicklung Spielraum lassen will. Mag noch bei der »Lebensbeendigung ohne den ausdrücklichen Willen des Patienten « die Skepsis überwiegen, so wird diese bald der Einsicht weichen, daß auch solche Fälle "gelöst " werden müssen.

Wirkliche Lösungen müssen in ganz anderer Hinsicht gefunden werden. Die erhebliche Ausweitung medizinisch-technischer 'Therapiemöglichkeiten bei gleichzeitiger Kostenexplosion hat zu einer neuen "Krise der Medizin " geführt. Der Ruf nach einer "Freigabe der direkten Euthanasie stellt insofern eine Gegenbewegung dar, welche den Menschen aber nicht ihren eigenen Tod zurückgeben kann. Sie verdeckt die eigentlichen Probleme, die das Lebensende eines jeden aufwerfen kann. Die moderne, funktionalistische Intensivmedizin hat eine KJuft aufgetan, indem sie den Menschen nur noch als ein beliebig austauschbares Objekt ihrer selbst betrachtet. Mangelhaft geschultes Personal, unzureichende Schmerztherapie, Anonymität und schließlich der "soziale Tod " ${ }^{\text {ral }}$ sind Faktoren, welche die Bcreitschaft sinken lassen, das Sterben anzunehmen, zu ertragen. Der geäußerte Stcrbewunsch hat sich oftmals als ein Ersuchen um mchr Sterbebeistand erwiesen '44, gewissermaßen um einen "Appell-Sterbewunsch “. Auf der Strecke bleibt dabei die Kunst der Sterbebegleitung, die "Ars moriendia, im is. Jahrhundert sogar eine eigene Literaturgattung. Mit einer verbesserten Betreuung Sterbender licße sich wahrscheinlich die Euthanasiediskussion insgesamt entschärfen, vielleicht gar überflüssig machen ${ }^{145}$.

Ist das menschliche Leben erst cinmal zur Verfügung gestellt, so ist seine Bcdeutung, ist sein Wert veränderbar und gesellschaftlichen Einflüssen unterworfen. Der Zweck der Beendigung auch unerträglichster Schmerzen darf niche das Mittel der Tötung heiligen. "Eine auf Mitleid allein gegründete Ethik ist etwas sehr Fragwürdiges... was sich da auftut für eine... Gewöhnung an den Gedanken und die Praxis des Tötens, das ist unabsehbar, «' ${ }^{146}$

$141 \mathrm{Zu}$ Gemeinsamkeiten und Unterschieden der zwanziger und dreißiger Jahre zu heutigen Tendenzen vgl. Rost, in: Bastian (Hrsg.), Denken - schreiben - toten, 1990, S. $37 \mathrm{ff}$

142 Bei Kuhse/Singer, should the baby live? « The Problem of Handicapped Infants, 1985, S. 192, ist dies bereits fur schwergeschadigte Neugeborene ausdrucklich der Fall.

143 Eser, JZ 1986, 786 (787).

144 v. Lutterotti, 1n: Eser (Hrsg.) (Fn. 2), S. 295; Sporken (Fn. 2), S. 279.

145 BGH JR 1993, 167 zeigt eınen kleinen Ausschnitt der unzulanglichen klinischen Zustande, die in diesem Fall zu Totungen aus Mitleid gefuhrt haben.

146 Hans Jonas, in: DIE ZEIT Nr. 35 vom 25. August 1989, S. I I. 